\title{
A PREDATOR-PREY SYSTEM INVOLVING GROUP DEFENSE: A CONNECTION MATRIX APPROACH
}

\author{
Konstantin Mischaikow $\dagger$ \\ Department of Mathematics, Michigan State University, East Lansing, MI 48824, U.S.A. \\ and \\ GaIL Wolkowicz $\ddagger$ \\ Department of Mathematics and Statistics, McMaster University, Hamilton, Ontario, Canada L8S $4 \mathrm{~K} 1$
}

(Received 13 January 1989; received for publication 15 September 1989)

Key words and phrases: Conley index, connection matrices, predator-prey system, group defense.

\section{INTRODUCTION}

THE PURPOSE of this paper is to give a concrete demonstration of how the connection matrix can be used to analyse a 1-parameter family of differential equations. In particular we have four goals:

(1) To show how to compute connection matrices.

(2) To use these matrices to classify the structure of solutions to our set of differential equations for various parameter values.

(3) To prove the existence of local and global bifurcations.

(4) To demonstrate how to ignore certain bifurcations.

Strange as it may seem, we want to emphasize the importance of this last goal. Even in seemingly simple systems it is possible for global bifurcations to occur which are difficult to detect or exclude (in our case, global bifurcations which lead to the existence of multiple periodic orbits). Nevertheless, one wants to be able to make significant statements about the general structure of the flow for all parameter values. Thus it is important to have techniques which are able to ignore subtle or difficult to detect phenomenon and still work.

The set of equations which we have chosen to study arise from a predator-prey model in which the prey exhibits group defense, i.e. the more prey the better their chances for protecting themselves from the predator. This set of equations has been studied before (see Freedman and Wolkowicz [3], Wolkowicz [13], and Mischaikow and Wolkowicz [9]) and we do not claim any new results, rather it is our techniques which are novel. To be more specific, the system we consider is two dimensional and hence phase plane techniques can be employed to obtain the results which we shall present herein. This is the approach taken in [13]. Of course, phase plane techniques are more difficult, if not impossible, to apply in the case of higher dimensional systems. The advantage of our approach is that the connection matrix is dimension independent and hence the tehniques we are describing here are in principle applicable to higher dimensional

\footnotetext{
$\dagger$ Research supported in part by NSF grant DMS-8507056. Current address: School of Mathematics, Georgia Institute of Technology, Atlanta, GA 30332, U.S.A.

$\ddagger$ Research supported by Natural Science and Engineering Research Council of Canada grant \#A9358 and by a Science and Engineering Research Board Grant from McMaster University.
} 
systems. This suggests to us that the connection matrix has the potential to be a powerful tool for studying multi-dimensional systems.

Because our example is planer, it is easy to visualize the dynamics which occur for the various parameter values. On the other hand, our approach is almost completely algebraic and hence it is easy to confuse what is intuitively clear with what we intend to prove. In fact the purpose of [9] is to demonstrate the relationship between the geometric properties of the dynamics in the phase plane and the algebraic quantities in our analysis. In this presentation we want to emphasize the power of the connection matrix techniques and, hence, we have been careful to avoid using the phase plane to obtain our results, though in some instances this would simplify our arguments.

Obviously one can not study differential equations from a purely algebraic point of view. At some point analytic arguments need to be introduced. In this paper we begin with such arguments, in particular we shall use 3 results of [13]:

(1) the set of bounded solutions is always compact;

(2) the structure of the bounded solutions when the parameter value is sufficiently large; and

(3) the eigenvalues of the linearized equations at critical points.

This is almost all the analysis we need and it is presented in Sections 2 and 3. To be more precise, in Section 2 we describe the 1-parameter family of equations which we shall study and the structure of the bounded solutions for large parameter values (theorem 2.1). In Section 3, in addition to giving a short introduction to Morse decompositions and connection matrices, we translate the above mentioned results of [13] into our terminology. As we shall see, to each Morse decomposition there corresponds a partial order and this partial order is crucial to the definition of a connection matrix. Thus the first computations we do are in Section 4 where we determine all the possible partial orders. Two points are worth mentioning; (i) we obtain our results from the information in Sections 2 and 3, and (ii) this is just a collection of possible urdetings, we du nut require that dii inese orderings be teailzed. Given the possioie partiai orderings, in Section 5 we compute the possible connection matrices. Again, at this stage we do not claim to have proven any existence results. Finally, in Section 6 we obtain the existence results, not only for the connection matrices (which imply certain "stable" dynamical structures) but also for the occurrence of bifurcations both local and global in nature. In addition we are able to give the order in which these bifurcations and connection matrices must occur. This information is summed up in a bifurcation graph (Fig. 1) which is obtained by comparing the connection matrices obtained in Section 5 and the knowledge of the connection matrix for large parameter values.

To summarize, we feel that our techniques are important because they are dimension independent and because they allow one to study parameterized families of differential equations given in some sense minimal information, such as the compactness of the set of bounded orbits and the eigenvalues of the linearized equations at critical points, information which is in principle obtainable even in more complicated higher dimensional systems.

\section{THE EQUATIONS AND THEIR BASIC PROPERTIES}

For a derivation of the following equations and an explanation of their biological relevance the reader is referred to $[3,9,13]$. We shall consider the following 1-parameter family of 
ordinary differential equations.

$$
\begin{array}{ll}
x^{\prime}=x g(x, K)-y p(x) \equiv p(x)(F(x, K)-y) \quad,=\mathrm{d} / \mathrm{d} t \\
y^{\prime}=y(-s+q(x))
\end{array}
$$

where $x, y \geq 0, s>0$ is a constant, and $K>0$, a constant, is the parameter value. $x(t)$ and $y(t)$ denote the density of prey and predator populations, respectively. In addition to the assumptions that follow we shall assume that the functions $g, p$ and $q$ are continuously differentiable.

The function $g(x, K)$ represents the growth rate of the prey in the absence of predation and for $x>0$ has the following properties:

$$
\begin{aligned}
g(K, K)= & 0, g(0, K)>0, g_{K}(x, K)>0, g_{x K}(x, K)>0, g_{x}(x, K)<0, \lim _{K \rightarrow \infty} g(0, K) \\
& \text { is finite, and } \lim _{K \rightarrow \infty} g_{x}(x, K)=0 .
\end{aligned}
$$

$p(x)$ is the predator response function and is assumed to satisfy:

$$
p(0)=0, p^{\prime}(0), p^{\prime \prime}(0)<0 \text {, and } p(x)>0 \text { for all } x>0 .
$$

In order to model the behavior of group defense we assume that there exists $h^{*}$ such that

$$
p^{\prime}(x)>0 \text { for } 0 \leq x<h^{*} \text { and } p^{\prime}(x)<0 \text { for } h^{*}<x .
$$

A final technical assumption is that

$$
p(x)-x p^{\prime}(x) \text { for all } x>0 .
$$

The rate of conversion of prey to predator is described by $q(x)$. In particular,

$$
\begin{aligned}
q(0)= & 0, q(x)>0 \text { for } x>0, q\left(h^{*}\right)>s, q^{\prime}(x)>0 \text { for } 0 \leq x<h^{*}, \text { and } q^{\prime}(x)<0 \\
& \text { for } x>h^{*} .
\end{aligned}
$$

The assumption that $q\left(h^{*}\right)>s$ implies the existence of $\lambda<h^{*}$ such that $q(\lambda)=s$. We shall also assume that there exists a $\mu>h^{*}$ such that $q(\mu)=s$. It is easy to check that this forces the predator isoclines to be the vertical lines $x=\lambda$ and $x=\mu$. The prey isocline is given by $F(x, K)=x g(x, K) / p(x)$.

There are at most four critical points of (2.1) lying in the first quadrant. The first two, $M(0)=(0,0)$ and $M(K)=(K, 0)$ always lie on the coordinate axis. The other two, $M(\lambda)=(\lambda, F(\lambda, K))$ and $M(\mu, F(\mu, K))$ exist inside the first quadrant if and only if $\lambda<K$ and $\mu<K$, respectively. If $\lambda=K$ (or $\mu=K$ ) then $M(\lambda)=M(K)$ (or $M(\mu)=M(K)$ ).

It is easy to see that the $x$-axis, $y$-axis and hence the interior of the first quadrant are all invariant under (2.1). Of course this is the biologically relevant set of initial conditions. However, for our analysis it proves useful to make the following assumptions on the flow outside of the first quadrant.

If $K<\lambda<\mu$ then $M(\lambda)$ is a saddle point, $M(\mu)$ is an attractor and the set of connections (see definition 3.1) $C(M(\lambda), M(K)$ ) and $C(M(\lambda), M(\mu)$ ) consist of unique orbits. Furthermore, these are the only bounded solutions outside of the closure of the first quadrant and they lie above the line $y=-L$ for some $L>0$.

If $\lambda<K<\mu$, then $M(\mu)$ is an attractor and $C(M(K), M(\lambda))$ consists of a unique orbit. Again this describes all the bounded solutions outside the first quadrant. We assume these bounded solutions all lie above the line $y=-L$.

We shall need the following result. 
THEOREM 2.1 (Wolkowicz [13]). Assume that for some $K^{\prime}>0, F$ has a local maximum $\left(x_{0}, F\left(x_{0}, K^{\prime}\right)\right)$. There exists $K^{*}>\mu$ such that if $K>K^{*}$, then all solutions of (2.1) with positive initial conditions converge to $M(K)$ except those originating on the stable manifold of $M(\mu)$ or at the point $M(\lambda)$.

\section{CONNECTION MATRICES}

We shall begin this section with the definitions of some basic ideas that are related to the connection matrix. For a more complete discussion of the Conley index the reader is referred to [ 1 , $11,12]$. For more details concerning the connection matrix and its properties see [2, 8, 10]. After this curt introduction we shall then return to the equations described in the previous section and state the results we need in order to compute connection matrices. The proofs of some of these results can be found in $[3,9,13]$.

Throughout this paper $S$ will always denote an isolated invariant set and $h(S)$, the Conley index of $S$.

Definition 3.1. Given two isolated invariant sets, $S$ and $S^{\prime}$, the set of connections from $S$ to $S^{\prime}$ is denoted by $C\left(S, S^{\prime}\right)=\left\{x \mid \omega(x) \subset S^{\prime}\right.$ and $\left.\omega^{*}(x) \subset S\right\}$ where $\omega(x)$ and $\omega^{*}(x)$ denote the omega and alpha limit sets of $x$, respectively.

A partially ordered set, $(P,>)$, consist of a finite set $P$ along with a strict partial order relation, $>$, which satisfies:

(i) $i>i$ never holds for $i \in P$, and

(ii) if $i>j$ and $j>k$, then $i>k$ for all $i, j, k \in P$.

An interval in $(P,>)$ is a subset, $I \subset P$, such that given $i, j \in I$, if $i<k<j$ then $k \in I$. The set of intervals in $(P,>)$ is denoted by $Q(P,>)$ or just $Q(>)$ if the set $P$ is fixed. An attracting inter$v a l, I \in \mathbb{D}(>)$, satisfies the additional condition that if $i \in I$ and $i>j$, then $j \in I$. A $(>)$ denotes the set of attracting intervals.

A Morse decomposition of $S$, denoted by $M(S)=\{M(i) \mid i \in(P,>)\}$, is a collection of mutually disjoint isolated invariant subsets of $S$, indexed by $(P,>)$, such that given $x \in S$, then either $x \in M(i)$ for some $i$, or there exists $i, j \in P$ such that $i>j$ and $x \in C(M(i), M(j))$. The invariant sets, $M(i)$, are called Morse sets.

Let $I \in O /(>)$, then one can define a new isolated invariant set

$$
M(I)=\left(\bigcup_{i \in I} M(i)\right) \cup\left(\bigcup_{j, i \in I} C(M(j), M(i))\right) .
$$

If $I \in \mathbb{A}$ then $M(I)$ is an attractor. Since $M(I)$ is an isolated invariant set $h(M(I))$ is defined. Let $H_{*}(I) \equiv H_{*}\left(h(M(I)) ; \mathbb{Z}_{2}\right)$, the singular homology of the pointed space $h(M(I))$. In particular, given $i \in P, H_{*}(i)=H_{*}\left(h(M(i)) ; \mathbb{Z}_{2}\right)$. If $\Delta: \underset{i \in P}{\oplus} H(i) \rightarrow \underset{i \in P}{\oplus} H(i)$ is linear, then $\Delta$ can be written as a matrix of maps $(\Delta(i, j))$ where $\Delta(i, j): H(i) \rightarrow H(j)$. Let $\Delta(I)=(\Delta(i, j))_{i, j \in I}$.

Definition 3.2. $\Delta$ is called a connection matrix for $M(S)$ if the following four properties are satisfied:

(i) If $i \geq j$ then $\Delta(i, j)=0$.

(ii) $\Delta(i, j) H_{k}(i) \subset H_{k-1}(j)$.

(strict upper triangularity)

(iii) $\Delta \cdot \Delta=0$.

(iv) $H(I) \approx$ kernel $\Delta(I) /$ image $\Delta(I)$ for every interval $I$. (degree - 1 map)

(rank condition) 
Theorem 3.3 (Franzosa [2]). Given a Morse decomposition there exists at least one connection matrix.

Notice that the partial order, $>$, plays an important role in the definition of a connection matrix. Therefore, given a fixed set $P$ with two different partial orderings one expects to obtain different connection matrices.

One of the most important properties of connection matrices is stated in the following theorem.

Theorem 3.4 (Franzosa [2]). If $\{i, j] \in 0(>)$ and if $\Delta(i, j)) \neq 0$, then $C(M(i), M(j)) \neq \varnothing$.

Because we are studying a two dimensional system, any heteroclinic orbit connecting two hyperbolic critical points with different index arises as the transverse intersection of the respective stable and unstable manifolds. Thus in our setting the following theorem is easy to apply. However, it can also be used in higher dimensional systems effectively. (See Hattori and Mischaikow [4].)

TheOREM 3.5 (McCord [6]). Let $M(S)=\{M(1), M(0) \mid 1>0\}$ be a Morse decomposition consisting of hyperbolic critical points. Assume

$$
H_{k}(i) \approx\left\{\begin{array}{cl}
\mathbb{Z}_{2} & \text { if } k=n+i \\
0 & \text { otherwise. }
\end{array}\right.
$$

Furthermore, let $C(M(1), M(0))$ consist of exactly $q$ heteroclinic orbits which arise as the transverse intersection of the stable and unstable manifolds of $M(0)$ and $M(1)$, respectively. Then $\Delta(1,0)=q \bmod 2$.

Because we are interested in studying a parametrized family of differential equations we need to know how connection matrices at different parameter values are related. This is the purpose of the following theorem.

THEOREM 3.6 (Mischaikow [8]). Let $S(K)$ be isolated invariant sets related by continuation for all parameter values $K>0$. If $\Delta_{K}$ and $\Delta_{K}$, are connection matrices for Morse decompositions of $S(K)$ and $S\left(K^{\prime}\right)$ respectively, then there exists a matrix, $T$, consisting of degree 0 maps, such that $\Delta_{K} T+T \Delta_{K},=0$.

The matrix $T$ is called a transition matrix and its entries give information about the existence of connecting orbits for the parameter values between $K$ and $K^{\prime}$.

We are now ready to relate these ideas to the predator-prey equations discussed in Section 2 . First, we define $S=$ (bounded solutions to (2.1)\}.

Proposition 3.7. $H_{*}\left(h(S) ; \mathbb{Z}_{2}\right)=(0,0, \ldots)$.

Proof. That all solutions to (2.1) with initial conditions in the first quadrant are bounded in forward time follows from a proof similar to the one given in Hsu [5, lemma 3.1]. Since this is dependent of $K$ and by our assumptions on the flow outside of the first quadrant, we have 
that $S$ is bounded for all $K$. Thus we can compute the index of $S$ by choosing a value of $K$ for which $S$ is contained in the first quadrant. As an isolating neighborhood of $S$ we choose $N=\left\{(x, y) \mid x^{2}+y^{2}=R, R\right.$ sufficiently large $\} \cap\{(x, y) \mid x \geq-\varepsilon$ and $y \geq-\varepsilon, \varepsilon>0$ but small $\}$. Given $N$ there exists an index pair, $\left(N_{1}, N_{0}\right)$, contained in $N$. For a more detailed description of $\left(N_{1}, N_{0}\right)$ see [9]. Notice that for $\varepsilon$ small, $\{(-\varepsilon, y) \in N\} \subset N_{0}$. Furthermore, $N_{0}$ can be chosen such that $\{(x,-\varepsilon) \in N \mid x>-\varepsilon\} \not \subset N_{0}$, and $N_{0}$ does not intersect the first quadrant (otherwise some orbit would be unbounded in positive time). Computing the homology of $N_{1} / N_{0}$ gives the desired result.

Obtaining a Morse decomposition of $S$ is slightly more subtle. It is clear that the critical points $M(K), M(0), M(\mu)$, and $M(\lambda)$ should be Morse sets, however it is possible that there are other invariant sets in $S$. To get around this problem we shall define another invariant set $M(\pi)$ which contains the "rest" of the invariant orbits.

Definition 3.8. $M(\pi)$ is defined to be the minimal isolated invariant set in the interior of the first quadrant which contains all periodic orbits of $S$.

Before we can discuss the properties of $M(\pi)$ we need the following information about the critical points of (2.1).

Proposition 3.9.

$$
\begin{aligned}
& H_{*}(0) \approx\left(0, \mathbb{Z}_{2}, 0,0, \ldots\right) \quad \text { for all } K>0 \text {. } \\
& H_{*}(K) \approx \begin{cases}\left(\mathbb{Z}_{2}, 0,0, \ldots\right) & \text { if } K<\lambda \text { or } \mu<K \\
\left(0, \mathbb{Z}_{2}, 0,0, \ldots\right) & \text { if } \lambda<K<\mu\end{cases} \\
& H_{*}(\lambda) \approx \begin{cases}\left(0, \mathbb{Z}_{2}, 0,0, \ldots\right) & \text { if } K<\lambda \\
\left(\mathbb{Z}_{2}, 0,0, \ldots\right) & \text { if } \lambda<K \text { and } F_{x}(\lambda, K)<0 \\
\left(0,0, \mathbb{Z}_{2}, 0, \ldots\right) & \text { if } \lambda<K \text { and } F_{x}(\lambda, K)>0\end{cases} \\
& H_{*}(\mu) \approx \begin{cases}\left(\mathbb{Z}_{2}, 0,0, \ldots\right) & \text { if } K<\lambda \\
\left(0, \mathbb{Z}_{2}, 0,0, \ldots\right) & \text { if } \mu<K .\end{cases}
\end{aligned}
$$

Proof. The proof follows from linearizing the equations about the various critical points. For details of the proof see [3,13].

We now return to the question of describing $M(\pi)$. Let $\gamma$ be the inner most periodic orbit in $M(\pi)$, if it exists. Then the compact region, $\Gamma$, bounded by $\gamma$ must contain at least one critical point. From (2.1) we have three possibilities $M(\lambda) \in \Gamma, M(\mu) \in \Gamma$, or $M(\lambda) \cup M(\mu) \subset \Gamma$. From proposition 3.9 , if $M(\mu)$ is in the interior of the first quadrant, then it is a saddle. This excludes $M(\mu) \in \Gamma$ and $M(\lambda) \cup M(\mu) \subset \Gamma$. Therefore, $M(\lambda) \in \Gamma$. Let $\gamma^{*}$ denote the outer most periodic orbit in $M(\pi)$, if it exists. A similar argument shows that if $\Gamma$ is the compact region bounded by $\gamma^{*}$ then, $M(\mu) \notin \Gamma$.

Proposition 3.10. If $\gamma$ and $\gamma^{*}$ exist then $\{M(p) \mid p \in P=\{0, K, \lambda, \mu, \pi\}\}$ is a set of isolated invariant subsets of $S$ and any other closed invariant subsets of $S$ intersects some $M(p)$ nontrivially. 
Proof. An equivalent statement of this proposition is that if $(x, y)$ lies on a bounded solution to (2.1) then $\omega(x, y) \subset M(p)$ and $\omega^{*}(x, y) \subset M\left(p^{\prime}\right)$ for some $p, p^{\prime} \in P$. Given our definition of $M(\pi)$ this is equivalent to the Poincaré-Bendixon theorem.

Remark 3.11. From now on whenever we refer to $M(\pi)$ we shall assume that $\gamma$ and $\gamma^{*}$ exist.

The collection $\{M(p) \mid p \in P\}$ will be taken to be the collection of Morse sets in our Morse decomposition of $S$. In the next section we shall determine what the appropriate partial orders are. However, we first need information about the index of $M(\pi)$.

Proposition 3.12.

(a) If $H_{*}(\lambda) \approx\left(\mathbb{Z}_{2}, 0,0, \ldots\right)$, then $H_{*}(\pi) \approx(0,0, \ldots)$ or $\left(0, \mathbb{Z}_{2}, \mathbb{Z}_{2}, 0,0, \ldots\right)$

(b) If $I_{*}(\lambda) \approx\left(0,0, \mathbb{Z}_{2}, 0,0, \ldots\right)$, then $H_{*}(\pi) \approx(0,0, \ldots)$ or $\left(\mathbb{Z}_{2}, \mathbb{Z}_{2}, 0,0, \ldots\right)$.

Proof. If $M(\pi)=\varnothing$ then $H_{*}(\pi) \approx(0,0, \ldots)$. So assume $M(\pi) \neq \varnothing$. To compute the index of $H_{*}(\pi)$ we need to construct an isolating neighborhood of $M(\pi)$. Let $N$ be a compact annulus containing $M(\pi)$ such that the inner ring, $R_{1}$, is arbitrarily close to $M(\lambda)$ and the outer ring, $R_{2}$, is arbitrarily close to $\gamma^{*}$. Notice that depending on the flow we can choose $R_{2}$ such that if $(x, y) \in \mathbb{R}_{2}$, then either $(x, y) \cdot t \in N$ for all $t>0$ or $(x, y) \cdot t \notin N$ for all $t>0$. Similarly, for $R_{1}$, however, in this case whether $R_{1}$ is an entrance or an exit set is determined by $M(\lambda)$. It is now a simple calculation to determine $H_{*}(\pi)$.

Proposition 3.13. If $(\lambda, \pi)$ is an attracting interval, then $H_{*}(\lambda \pi) \approx\left(\mathbb{Z}_{2}, 0,0, \ldots\right)$.

Proof. We can define an isolating neighborhood for $M(\lambda \pi)$ as follows. Choose $R_{2}$ as in the proof of proposition 3.12. Let $N$ be the compact region bounded by $R_{2}$. Since $M(\lambda \pi)$ is an attractor, $R_{2}$ is an entrance set for $N$, i.e. the exit set for $N$ is the empty set. Clearly, $H_{*}\left(N, \varnothing ; \mathbb{Z}_{2}\right) \approx H_{*}\left(\lambda \pi \approx\left(\mathbb{Z}_{2}, 0,0, \ldots\right)\right.$.

Proposition 3.14. Assume $M(\pi) \neq \varnothing$ and $\lambda>\pi$, then $H_{*}(\lambda) \approx\left(0,0, \mathbb{Z}_{2}, 0,0, \ldots\right)$.

Proof. $M(\pi) \neq \varnothing$ implies that $\lambda \in \Gamma$. This in turn implies that $\lambda$ lies in the interior of the first quadrant and hence that $\lambda<K$. However, in the partial order $\lambda>\pi$ and hence $M(\lambda)$ is not an attractor. The result now follows from proposition 3.9 .

\section{PARTIAL ORDERS}

Theorem 3.3 states that for every Morse decomposition of $S$ there exists at least one connection matrix. However, a Morse decomposition consists not only of a collection of Morse sets, but also of a partial ordering for these sets. Thus, before we can consider the question of what are the connection matrices associated to (2.1) we need to determine the set of partial orderings.

From the previous section we know that $P=\{O, \lambda, \mu, K, \pi\}$ can be used as an indexing set for a Morse decomposition as long as we realize that in some cases $M(\pi)=\varnothing$. Therefore, we must determine the admissible partial orders on $P$ as a function of $K$. 
Proposition 4.1. The set of admissible partial orderings on $P$ is as follows:

(a) If $K<\lambda<\theta$, define

$$
>_{1} \quad \text { by } O>_{1} p, \lambda>_{1} K, \lambda>{ }_{1} \mu .
$$

(b) If $\lambda<K<\mu$, define

$$
\begin{aligned}
& >_{2} \quad \text { by } O>_{2} p, K>_{2} \mu, K>_{2} \pi, \pi>_{2} \lambda \\
& >_{3} \quad \text { by } O>_{3} p, K>_{3} \mu, K>_{3} \pi, \lambda>_{3} \pi .
\end{aligned}
$$

(c) If $\lambda<\mu<K$, defïne

$$
\begin{array}{ll}
>_{4} & \text { by } O>_{4} p, \mu>_{4} K, \mu>_{4} \pi, \pi>_{4} \lambda \\
>_{5} & \text { by } O>_{5} p, \mu>_{5} K, \pi>_{5} \mu, \pi>_{5} \lambda \\
>_{6} & \text { by } O>_{6} p, \mu>_{6} K, \lambda>_{6} \pi, \mu>_{6} \pi \\
>_{7} & \text { by } O>_{7} p, \mu>_{7} K, \lambda>_{7} \pi, \pi>_{7} \mu
\end{array}
$$

where $p \in P$. Furthermore, $\{O, K\},\{\lambda, \pi\} \in I\left(>_{j}\right)$ for all $j$.

The following lemmas shall prove useful in the proof of this proposition.

Lemma 4.2. $O>p$ is an admissible ordering for all values of $p \in P \backslash\{O\}$. Furthermore, $\{O, K\} \in \mathbb{I}$.

Proof. $M(O)$ is a saddle with the $y$-axis as its stable manifold, hence $C(M(p), M(O))=\varnothing$ for all $p \in P$ thus we can assume that $O>p$. The unstable manifold of $M(O)$ is the $x$-axis and $M(K)$ is the only invariant set on the $x$-axis (as long as $K \neq \lambda, \mu)$. Thus $\{O, K\} \in \vdots$.

LEMMA 4.3. If $K<\lambda<\mu$ or $\lambda<\mu<K$ then $p>K$ is an admissible ordering for all values of $p \in P \backslash\{K\}$.

Proof. For these parameter values $K$ is an attracting fixed point hence

$$
C(M(K), M(p))=\varnothing .
$$

Lemma 4.4. If $K<\mu$ then $p>\mu$ is an admissible ordering for all values of $p \in P \backslash\{\mu\}$.

Proof. For these parameter values $M(\mu)$ is an attracting fixed point.

Proof of proposition 4.1. Case (a). From a previous proposition we know that $M(\pi)=\varnothing$, hence $\pi$ need not be related to $O, \lambda, \mu$, or $K$. The result now follows from lemmas 4.2, 4.3, and 4.4 .

Case (b). It is easy to check that the stable manifold for $M(K)$ consists of the positive $x$-axis, thus $C(M(p), M(K))=\varnothing$ for $p \in P \backslash\{O\}$. In particular, without loss of generality we can assume that $K>\mu$ and $K>\pi$. Combining this with lemma 4.2 gives $>_{2}$ and $>_{3}$ as the only remaining possible partial orders.

Case (c). Use lemmas 4.2 and 4.3 and write down the remaining possible partial orders.

That $\{\lambda, \pi\} \in \mathbb{Q}\left(>_{i}\right)$ follows from the discussion concerning $M(\pi)$ preceding proposition 3.10 . 


\section{CONNECTION MATRICES}

We are now in the position to be able to compute all the possible connection matrices of our parameterized system of equations. Let $\Delta^{i}$ denote the connection matrix associated to the Morse decomposition $M(S)=\left\{M(p) \mid p \in\left(P,>_{i}\right\}\right.$.

LEMMA 5.1. If $H_{*}(K) \approx\left(\mathbb{Z}_{2}, 0,0, \ldots\right)$, then $\Delta(O, K)=1$.

Proof. Apply theorem 3.5.

Proposition 5.2.

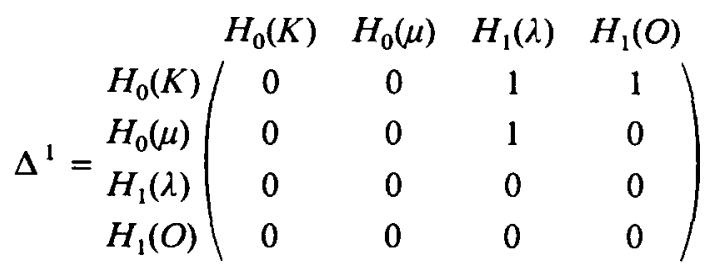

Proof. The first thing to notice is that since $K<\lambda, M(\lambda)$ does not lie in the first quadrant and hence $M(\pi)=\varnothing$.

By definition $\Delta$ is upper triangular. Furthermore, since connection matrices are degree-1 maps the only possible nonzero entries are $\Delta(i, j)$ where $i=\lambda$ or $O$ and $j=K$ or $\mu$. By lemma $5.1 \Delta(O, K)=1$. By our assumptions on the flow outside of the positive orthant, $\Delta(\lambda, K)=\Delta(\lambda, \mu)=1$. Finally, since $H_{k}(h(S))=0$ for all $k$, the rank of $\Delta$ is 2 and hence, $\Delta(O, \mu)=0$.

Proposition 5.3.

where $a+b=1$.

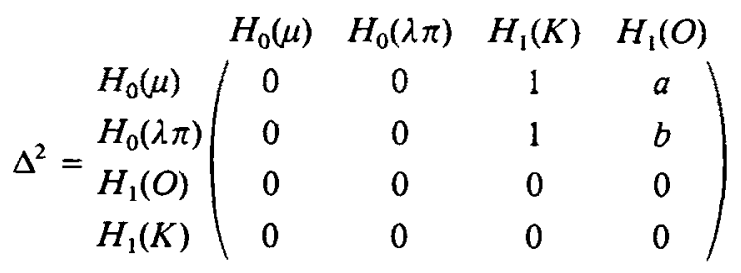

Proof. Notice that $\lambda \pi \in \mathbb{A}\left(>_{2}\right)$. Therefore, by proposition $3.11 H_{*}\left(\lambda \pi \approx\left(\mathbb{Z}_{2}, 0,0, \ldots\right)\right.$.

Since $\Delta$ is a degree -1 upper triangular matrix we only need to examine the $\Delta(i, j)$ entries where $i=K$ or $O$ and $j=\mu$ or $\lambda . \Delta(K, \mu)=1$ by our assumptions on the flow outside of the positive orthant. The other branch of the unstable manifold of $K$ lies inside the positive orthant and hence is bounded. In particular it must limit to $M(\lambda \pi)$. Since this is the unique connecting orbit between $M(K)$ and $M(\lambda \pi)$, by theorem $3.5 \Delta(K, \lambda \pi)=1$. There is no connecting orbit from $M(O)$ to $M(u)$ or $M(\lambda \pi)$, however, they are not adjacent in the partial order hence the corresponding entries in the connection matrix are not flow defined. Since $H_{k}(h(S))=0$ for all $k$, the rank of $\Delta$ is two and hence $a+b=1$.

COROLLARY 5.4. If $>_{2}$ is an admissible partial ordering, then $H_{*}(\pi)=0$. 
Remark. There is no unique connection matrix given the partial order $>_{2}$. A heuristic explanation for this fact is that connection matrices continue under perturbation. Since the connection from $M(O)$ to $M(K)$ is not transverse one expects that a generic perturbation of our equation would lead to either an $M(O)$ to $M(\mu)$ or an $M(O)$ to $M(\lambda \pi)$ connection appearing. In the first case $a=1$ and $b=0$ and in the second case $a=0$ and $b=1$. Because the explicit values of $a$ and $b$ will not play a role in our latter analysis we shall treat $\Delta^{2}$ as if it were one matrix rather than two. We shall do the same for $\Delta^{3}$ defined below.

LEMMA 5.5. If $C(M(K), M(\pi))$ consists of a unique orbit and $H_{*}(\pi) \approx\left(\mathbb{Z}_{2}, \mathbb{Z}_{2}, 0, \ldots\right)$, then $\Delta(K, \pi)=1$.

Proof. Choose an isolating neighborhood, $N$, for $M(\pi)$ as in proposition 3.12. Let $B$ be a small disk containing $M(K)$, then $B$ is an isolating neighborhood for $M(K)$. Finally, let $\Sigma \subset B$ be a transverse section to the connecting orbit from $M(K)$ to $M(\pi)$. Then $N \cup B \cup \Sigma \cdot[0, \infty)$ is an isolating neighborhood for $M(K \pi)$. One can use this to check that $H_{*}(K \pi) \approx 0$.

Proposition 5.6.

where $a+b=1$.

\begin{tabular}{|c|c|c|c|c|c|c|}
\hline & $H_{0}(\mu)$ & $H_{0}(\pi)$ & $H_{1}(\pi)$ & $H_{1}(K)$ & $H_{1}(O)$ & $H_{2}(\lambda)$ \\
\hline$H_{0}(\mu)$ & 0 & 0 & 0 & 1 & $a$ & 0 \\
\hline$H_{0}(\pi)$ & 0 & 0 & 0 & 1 & $b$ & 0 \\
\hline$=H_{1}(\pi)$ & 0 & 0 & 0 & 0 & 0 & 1 \\
\hline$H_{1}(K)$ & 0 & 0 & 0 & 0 & 0 & 0 \\
\hline$H_{1}(O)$ & 0 & 0 & 0 & 0 & 0 & 0 \\
\hline$H_{2}(\lambda)$ & 0 & 0 & 0 & 0 & 0 & 0 \\
\hline
\end{tabular}

Proof. We begin by showing that $H_{2}(\lambda)=\mathbb{Z}_{2}$. By proposition 3.14 this is equivalent to showing that $M(\pi) \neq \varnothing$. So assume that $M(\pi)=\varnothing$. Notice that we can now let $\pi>\lambda$, since $M(\pi)$ does not really exist. This puts us back into the partial order $>_{2}$. So without loss of generality we can choose $H_{2}(\lambda) \approx \mathbb{Z}_{2}$.

We now leave it to the reader to check that if $H_{*}(\pi) \approx 0$ then one cannot construct a connection matrix (the rank condition will fail). Thus by proposition $3.12, H_{*}(\pi) \approx\left(\mathbb{Z}_{2}, \mathbb{Z}_{2}, 0, \ldots\right)$.

$\Delta$ is a degree -1 upper triangular matrix, therefore, we need only consider the following entries: $\Delta(\pi, \mu), \Delta(K, \mu), \Delta(K, \pi), \Delta(O, \mu), \Delta(O, \pi), \Delta(\lambda, \pi)$ and $\Delta(\lambda, K) .\{\lambda, K\} \in \mathbb{Q}\left(>_{3}\right)$ and $C(M(\lambda), M(K))=\varnothing$, thus by theorem $3.4 \Delta(\lambda, K)=0$. The rank of $\Delta$ equals 3 , Hence $\Delta(\lambda, \pi)=1$. Recall that by definition $\Delta \cdot \Delta=0$. This forces $\Delta(\pi, \mu)=0$. The same argument as in the proof of proposition 5.2 shows that $\Delta(K, \mu)=1 . \Delta(K, \pi)=1$ by lemma 4.3 . Finally, the rank condition forces $a+b=1$.

Proposition 5.7.

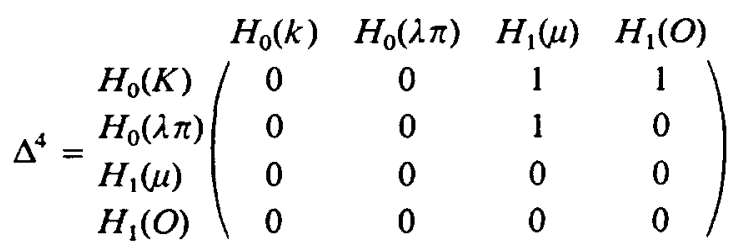


Proof. Since $\{\lambda \pi\} \in \mathbb{A}\left(>_{4}\right)$, by proposition $3.13, H_{*}(\lambda \pi) \approx\left(\mathbb{Z}_{2}, 0,0, \ldots\right)$. The rest of the argument is similar to that of proposition 5.2.

Proposition 5.8.

$$
\begin{aligned}
& \begin{array}{llllll}
H_{0}(K) & H_{0}(\lambda) & H_{1}(\mu) & H_{1}(O) & H_{l}(\pi) & H_{2}(\pi)
\end{array} \\
& \left.\Delta^{5}=\begin{array}{c|cccccc}
H_{0}(K) & 0 & 0 & 0 & 1 & * & 0 \\
H_{0}(\lambda) & 0 & 0 & 0 & 0 & 1 & 0 \\
H_{1}(\mu) & 0 & 0 & 0 & 0 & 0 & 1 \\
H_{1}(O) & 0 & 0 & 0 & 0 & 0 & 0 \\
H_{1}(\pi) & 0 & 0 & 0 & 0 & 0 & 0 \\
H_{2}(\pi) & 0 & 0 & 0 & 0 & 0 & 0
\end{array}\right)
\end{aligned}
$$

where * denotes an undetermined entry.

Proof. To determine $H_{*}(\pi)$ and $H_{*}(\lambda)$ notice that $\pi>s \lambda$ and apply an argument similar to that of proposition 5.6.

Since $\Delta$ is a degree-1 upper triangular matrix we need only consider the following entries: $\Delta(\mu, K), \Delta(\mu, \lambda), \Delta(O, K), \Delta(O, \lambda), \Delta(\pi, K), \Delta(\pi, \lambda)$, and $\Delta(\pi, \mu)$. The rank of $\Delta$ is 3 , hence $\Delta(\pi, \mu)=1$. This and the fact that $\Delta \circ \Delta=0$ implies that $\Delta(\mu, K)=\Delta(\mu, \lambda)=0$. Proposition 5.1 implies that $\Delta(O, K)=1$. One can check that $\{O, \lambda\} \in \square\left(>_{s}\right)$. Since $C(M(O), M(\lambda))=\varnothing$ it now follows that $\Delta(O, \lambda)=0$. The rank condition now requires $\Delta(\pi, \lambda)=1$.

It is worth noting that a more careful analysis of the flow will uniquely determine the unknown entry in $\Delta^{5}$, however, for our analysis this is not necessary. The point to be made here is that connestion matrices can be useful even if thoy ane not complately detcrmined.

Proposition 5.9.

$$
\begin{aligned}
& \begin{array}{llllll}
H_{0}(K) & H_{0}(\pi) & H_{1}(\pi) & H_{1}(\mu) & H_{1}(O) & H_{2}(\lambda)
\end{array} \\
& \left.\Delta^{6}=\begin{array}{l|llllll}
H_{0}(K) & 0 & 0 & 0 & 1 & 1 & 0 \\
H_{0}(\pi) & 0 & 0 & 0 & 1 & 0 & 0 \\
H_{1}(\pi) & 0 & 0 & 0 & 0 & 0 & 1 \\
H_{1}(\mu) & 0 & 0 & 0 & 0 & 0 & 0 \\
H_{1}(O) & 0 & 0 & 0 & 0 & 0 & 0 \\
H_{2}(\lambda) & 0 & 0 & 0 & 0 & 0 & 0
\end{array}\right)
\end{aligned}
$$

Proof. The proof is similar to that of proposition 5.8 .

Proposition 5.10.

$$
\Delta^{7}=\begin{gathered}
H_{0}(K) \\
H_{0}(K) \\
H_{1}(\mu) \\
H_{1}(O) \\
H_{2}(\lambda)
\end{gathered}\left(\begin{array}{cccc}
0 & 0 & 1 & 0 \\
0 & 0 & 0 & 1 \\
0 & 0 & 0 & 0 \\
0 & 0 & 0 & 0
\end{array}\right)
$$


Proof. By proposition 3.14, $H_{*}(\lambda) \approx\left(0,0, \mathbb{Z}_{2}, 0, \ldots\right)$. However, $\pi>_{7} \mu$, also, and hence $H_{*}(\pi) \approx 0$.

The only possible nonzero entries are $\Delta(\mu, K), \Delta(O, K)$, and $\Delta(\lambda, \mu)$. Lemma 5.1 tells us that $\Delta(O, K)=1$. The rank condition forces $\Delta(\lambda, \mu)=1$ and finally, $\Delta \circ \Delta=0$ implies that $\Delta(\mu, K)=0$.

\section{BIFURCATIONS}

Notice that we have not defined connection matrices for all possible values of $K$. This is because if $K=\lambda$ or $K=\mu$ then we are at a bifurcation point. In this section we shall use the connection matrices we obtained in Section 5 to describe the order in which bifurcations can occur and how the structure of the invariant sets must change. Once again, it must be emphasized that at this point the matrices in Section 5 are only known to be the possible connection matrices. We still have not proved which of them actually occur. However, by the end of this section (theorem 6.7) we will be able to give some existence results.

Because of the structure of the equations which we are studying, there are only certain bifurcations which can occur as $K$ is changed. In particular, $M(O)$ undergoes no bifurcations, $M(K)$ and $M(\mu)$, and $M(K)$ and $M(\lambda)$ can exchange stability. $M(\lambda)$ can undergo a Hopf bifurcation. Globally, $M(\mu)$ may have a homoclinic orbit associated with it. Finally, the periodic orbits themselves may be subject to bifurcations (i.e. saddle node, pitchfork, etc.). Given the way we have defined $M(\pi)$ we cannot detect this latter set of bifurcations and so we shall intentionally ignore them. This does not mean that we are assuming that they do not occur, but rather that our analysis is valid regardless of whether such bifurcations are occurring or not.

Since we now know all the possible connection matrices for those values of $K$ at which a bifurcation is not occurring we shall adopt the following approach. Given $\Delta^{i}$, we determine which of the four elementary bifurcations, $\lambda$-exchange $(M(\lambda)$ and $M(K)$ exchange stability), $\mu$ exchange $(M(\mu)$ and $M(K)$ exchange stability), Hopf $(M(\lambda)$ undergoes a Hopf bifurcation), or homoclinic $(M(\mu)$ is the critical point on a homoclinic orbit), is possible and then given this elementary bifurcation what is the new connection matrix.

Our results can be most clearly presented if we make the following "generic" assumption. Let the set of points at which the elementary bifurcations occur be a discrete set and assume that at each bifurcation point only one elementary bifurcation occurs.

Lemma 6.1. There exists $K$ such that if $K>K^{*}$ then the connection matrix is $\Delta^{7}$.

Proof. From Wolkowicz [13], theorem 3.7 we have that there exists a $K^{*}>\mu$ such that if $K>K^{*}$, then all solutions of $(2.1)$ with initial conditions in the positive orthant converge to $M(K)$ except those originating on the stable manifold of $M(\mu)$ or at the point $M(\lambda)$. Since $K^{*}>\mu$ the connection matrix must be $\Delta^{i}$ where $i=4,5,6$, or 7 . By looking at the nonzero entries of all these matrices and using theorem $3.4, \Delta^{i}$ for $i=4,5$, or 6 is eliminated. Since a connection matrix exists it must be $\Delta^{7}$.

We begin with the case where $K<\lambda$. From propositions 4.1 and 5.2 it is clear that the connection matrix is $\Delta^{1}$. Thus if a bifurcation occurs at $K^{\prime}<\lambda$ then this bifurcation cannot result in a change in the connection matrix. This rules out a $\lambda$ or $\mu$-exchange since this would result in different values for $H(\lambda), H(\mu)$ or $H(K)$. Similarly, a Hopf bifurcation cannot occur. Finally, since we are only interested in the bifurcations that occur in the closure of the first orthant we 
can eliminate the occurrence of a homoclinic orbit. Therefore, no bifurcation can occur for $K<\lambda$. This means that the first bifurcation occurs at $K=\lambda$ where a $\lambda$-exchange takes place.

LEMMA 6.2. $\Delta^{1} \stackrel{\lambda}{\longrightarrow} \Delta^{2}$.

Before we prove this lemma a word of explanation may be in order. What is meant by the above notation is that passing through a $\lambda$-exchange bifurcation results in a change of connection matrices between $\Delta^{1}$ and $\Delta^{2}$. Furthermore, the arrow indicates that as $K$ increases through the $\lambda$-exchange bifurcation point, it is not possible to change from $\Delta^{2}$ to $\Delta^{1}$.

Proof of lemma 6.2. We are given that $K<\lambda$ implies that the connection matrix is $\Delta^{\prime}$. Also, by proposition 4.1 for $\lambda<K<\mu$ the only possible connection matrices are $\Delta^{2}$ and $\Delta^{3}$. Given that only a $\lambda$-exchange occurs, checking $H_{*}(K)$ and $H_{*}(\lambda)$ implies the result.

We now consider what can happen for the parameter values $\lambda<K<\mu$. As was mentioned before, the only possible connection matrices are $\Delta^{2}$ and $\Delta^{3}$ and again arguing as above this excludes the possibility of a $\lambda$ or $\mu$-exchange. Since $\lambda$ is the only critical point in the positive orthant a homoclinic orbit is impossible (since we are in the plane the homoclinic orbit would have to surround another critical point). This only leaves the possibility of a Hopf bifurcation at $M(\lambda)$. Assume then that a Hopf bifurcation takes place at $K^{\prime} \in(\lambda, \mu)$. By lemma 6.2 we know that if $K \in\left(\lambda, K^{\prime}\right)$ then the connection matrix is $\Delta^{2}$. Thus, for $K \in\left(K^{\prime}, \mu\right)$, we have that $H_{2}(\lambda)=\mathbb{Z}_{2}$. Therefore the connection matrix is $\Delta^{3}$ and we have the following lemma.

LEMMA 6.3. $\Delta^{2} \stackrel{H}{\longrightarrow} \Delta^{3}$.

The fact that the Hopf bifurcation is directed follows from the linear analysis and the assumption on $p(x)$.

LEMMA 6.4. $\Delta^{2} \underset{\mu}{\longrightarrow} \Delta^{4}$ and $\Delta^{3} \underset{\mu}{\longrightarrow} \Delta^{6}$.

Proof. We have already established that for $K \in(\lambda, \mu)$ the only bifurcation that can occur is a Hopf bifurcation. Therefore, the $\mu$-exchange must occur when $K=\mu$. By proposition 4.1 if $\mu<K$ then the set of possible connection matrices are $\Delta^{i}$ where $i=4,5,6$, or 7 . The same type of argument as that used in lemma 6.2 gives the desired result.

We are now in the region $\mu<K$. An argument similar to that which lead to lemma 6.3 gives:

LEMMA $6.5 . \Delta^{4} \stackrel{H}{\longrightarrow} \Delta^{6}$ and $\Delta^{5} \stackrel{H}{\longrightarrow} \Delta^{7}$.

We still need to determine what bifurcation occurs when the connection matrices change from $\Delta^{4}$ or $\Delta^{6}$ to $\Delta^{5}$ or $\Delta^{7}$.

LEMMA 6.6. $\Delta^{4} \stackrel{h}{\longrightarrow} \Delta^{5}$ and $\Delta^{6} \stackrel{h}{\longrightarrow} \Delta^{7}$.

Proof. We shall prove the first diagram, noting that the second result follows from a similar argument. From theorem 3.6 we know that there exists a transition matrix $T$ satisfying the 
equation $\Delta^{4} T+T \Delta^{5}=0$. Since $T$ is a degree 0 map it takes the form

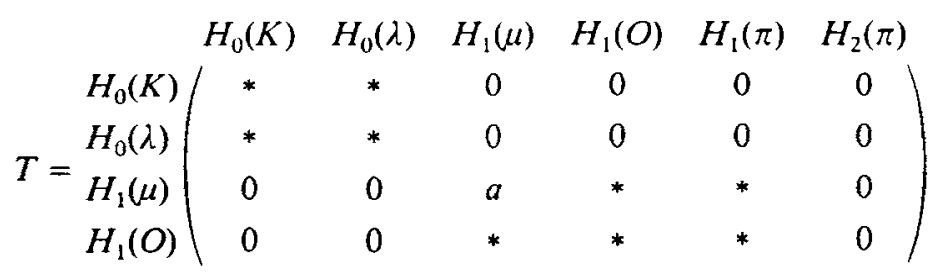

In order for the above mentioned equation to be satisfied, $T(\mu, \mu)=a=0$. Now by Mischaikow [7, corollary 4.4] there exists a homoclinic orbit to $M(\mu)$.

We can collect the information of the previous lemmas and form the bifurcation graph associated to this parameterized family of equations as is done in Fig. 1. As can be seen the vertices of this graph consist of the connection matrices from Section 5 and the edges are made up of the possible bifurcations. Using this information we can obtain the following results.

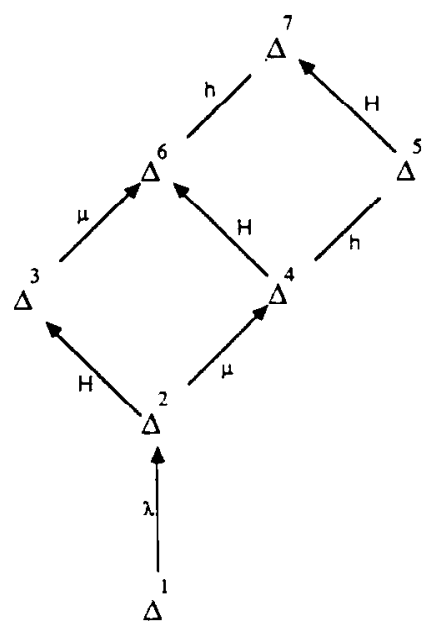

Bifurcation Graph

Fig. 1.

THEOREM 6.7.

(a) There exists a unique value for which a $\lambda$-exchange occurs.

(b) There exists a unique value for which a $\mu$-exchange occurs.

(c) There exists a unique value for which a Hopf bifurcation occurs.

(d) An odd number of homoclinic bifurcations always occurs.

(e) For a particular choice of functions $p, g$, and $q$, the connection matrices which are realized are determined by the relative values of $K$ for which the Hopf bifurcation occurs.

(f) For any choice of $p, g$, and $q$, there exist values of $K$ such that $\Delta^{1}, \Delta^{2}$, and $\Delta^{7}$ are connection matrices.

(g) For a particular choice of functions $p, g$, and $q$, it is not possible for both $\Delta^{3}$ and $\Delta^{4}$ to be connection matrices for various parameter values. 
(h) If for a particular choice of functions $p, g$, and $q$, there exist parameter values $K_{5}$ and $K_{6}$ for which $\Delta^{5}$ and $\Delta^{6}$ are connection matrices, respectively, then $K_{5}<K_{6}$.

Proof. Beginning with $K$ small and proceeding to $K$ large we are forced to travel a path through the bifurcation graph which begins at $\Delta^{1}$ and ends at $\Delta^{7}$ (lemma 6.1). Furthermore, we can only travel on the edges in the direction in which the arrows are pointing.

\section{REFERENCES}

1. Conley C. C., Isolated invariant sets and the morse index, CBMS Regional Conf. Series in Math. 38. AMS, Providence, Rhode Island (1978).

2. Franzosa R., The connection matrix theory for Morse decompositions, Trans. Am. math. Soc. 311, 561-592 (1989).

3. Freedman H. I. \& Wotkowicz G. S. K., Predator-prey systems with group defense: the paradox of enrichment revisited, Bull. math. Biol. 48, 493-508 (1986).

4. HatToRI H. \& Mischaikow K., On the existence of intermediate magnetohydrodynamic shock waves (to appear).

5. Hsu S. B., On global stability of a predator-prey system, Math. Biosci. 39, 1-10 (1978).

6. McCord C., The connection map for attractor-repeller pairs, Trans. Am. math. Soc. 307, 195-203 (1988).

7. Mischaikow K., Existence of generalized homoclinic orbits for one-parameter families of flows, Proc, Am. math. Soc. 103, 59-68 (1989).

8. Mischaikow K., Transition matrices, Proc. R. Soc. Edinb. 112A, 155-175 (1989).

9. Mischaikow K. \& Wolkowicz G. S. K., A connection matrix approach illustrated by means of a predator-prey model involving group defense, 1986 Proc. of Trieste Research Conf. on Mathematical Ecology, (Edited by T. G. Hallam, L. J. Gross, and S. A. Levin), World Scientific Publishing, 676-710 (1988).

10. ReINECK J., Connecting orbits in one-parameter families of flows, Engng Theor. Dyn. Syst. 8*, 359-374 (1988).

11. Salamon D., Connected simple systems and the Conley index of isolated invariant sets, Irans. Am. math. Soc. 291, $1-41$ (1985).

12. SmOLLer J., Shock Waves and Reaction Diffusion Equations. Springer, New York (1983).

13. WoLkowicz G. S. K., Bifurcation analysis of a predator-prey system involving group defense, SIAM J. Appl. Math. 48, 1-15 (1988). 